\title{
ANALYSIS OF FLOW BEHAVIOUR AND CHARACTERISTICS OF PNEUMATIC COMPONENTS
}

\author{
Daniel HUBERT *, Sylvie SESMAT**, Rosario De GIORGI**, Didier GAUTIER*** and \\ Eric BIDEAUX** \\ * ASCO JOUCOMATIC NUMATIC \\ 32 Avenue Albert 1er, BP 312, 92506 Rueil Malmaison Cedex, France \\ (E-mail: daniel.hubert@emerson.com) \\ ** Laboratoire AMPERE, INSA-Lyon (UMR CNRS 5005) \\ 25 avenue Jean Capelle, 69621 Villeurbanne Cedex, France \\ (E-mail: sylvie.sesmat@insa-lyon.fr) \\ *** CETIM, 52 avenue Félix Louat, BP 80067 \\ 60304 Senlis Cedex, France
}

\begin{abstract}
Information concerning the flow characteristics of pneumatic components is essential not only for selecting the right component at the design stage, but also for the simulation and the validation of different performances of a circuit. Recently, some works concerning the revision of the ISO 6358:1989 standard [1] have concerned both the mathematical approximation of the mass flow rate characteristic [2] and the experimental way to obtain the characteristics [3, 4, 5]. This paper proposes to discuss the importance of the upstream and downstream pressures taken as references. Using experimental results, the flow behaviour of two real components is discussed pointing out two different cases, the first being close to a convergent nozzle, the second to a convergent-divergent nozzle.
\end{abstract}

\section{KEY WORDS}

Mass flow rate characterisation, convergent behaviour, convergent-divergent behaviour, pressure measurement

\section{NOMENCLATURE}

$\begin{aligned} A: & \text { geometrical area } \\ C: & \text { conductance } \\ p: & \text { pressure } \\ p_{b}: & \text { back pressure } \\ p_{e}: & \text { exit pressure } \\ q_{m}: & \text { mass flow rate } \\ R: & \text { gas constant } \\ T: & \text { temperature } \\ u: & \text { velocity } \\ \gamma: & \text { specific-heat ratio }(1.4 \text { for air }) \\ \rho: & \text { density }\end{aligned}$

$\left[\mathrm{m}^{2}\right]$
$\left[\mathrm{m}^{3} / \mathrm{s} / \mathrm{Pa}\right]$
$[\mathrm{Pa}]$
$[\mathrm{Pa}]$
$[\mathrm{Pa}]$
$[\mathrm{kg} / \mathrm{s}]$
$[\mathrm{J} /(\mathrm{kg} \cdot \mathrm{K})]$
$[\mathrm{K}]$
$[\mathrm{m} / \mathrm{s}]$
$\left.\left[\mathrm{kg} / \mathrm{m}^{3}\right)\right]$

\author{
subscripts \\ $c:$ critical \\ $d$ : port component diameter \\ $D$ : largest diameter $\mathrm{D}$ \\ ISO: $\quad$ reference to ISO6358 standard [1] \\ $t:$ total \\ 0 : stagnation conditions \\ 1 : upstream conditions \\ 2 : downstream conditions

\section{INTRODUCTION}

ISO6358:1989 standard [1] defines both mathematical approximation of the mass flow rate characteristic and the experimental way to obtain the characteristics. This 
standard provides an efficient help to manufacturers to characterise the flow capacity of pneumatic components with a couple of parameters: the sonic conductance $C$ and the critical pressure ratio $b$. Therefore, this gives very useful information to circuit designers for the component choice. This standard is under revision because it requires some improvements into three directions.

First, the use of two additional parameters has recently extended the range of application of the standard to new components [2]. Second, two new experimental methods for characterizing the components have been proposed. They are based on transient characterisations (discharge method [3, 4] or charge method [5]). But third, whatever the experimental method is, a study has to be carried out in the light of fluid mechanic theory in order to determine the appropriate location of the pressure sensors used for the measurement of the upstream and downstream pressures. This is the purpose of this paper.

After a brief summary of fluid mechanic theory about flow in convergent and convergent-divergent nozzles, different measurement results are discussed according to the way upstream and downstream pressures are measured. For this study two spool valves are characterised. Component 1 has G1/8 connecting ports while component 2 has G1/4.

\section{FLUID MECHANICS MODELING}

Up to now, most of pneumatic flow models relies on the theoretical results obtained in fluid dynamics for an ideal convergent nozzle $[6,7]$. The case of ideal convergent-divergent nozzle is also interesting. The modelling considers that the flow is one dimensional, isentropic (adiabatic and reversible) and stationary, and that friction forces are negligible [7]. There is an upstream large reservoir with a stagnation pressure $p_{0}$ and a stagnation temperature $T_{0}$. The flow results from a back pressure $p_{b}$ (downstream and outside the convergent nozzle) lower than $p_{0}$.

The continuity equations (mass and energy) are used to determine theoretically the pressure distributions and the mass flow values according to the pressure conditions $\left(p_{0}, p_{b}\right)$.

\section{Flow behaviour in a convergent nozzle}

Considering the ideal nozzle (Fig.1), the fluid mechanic theory points out two flow behaviours delimited by the critical pressure $p_{c}$ at which the throat becomes sonic: a subsonic flow and a sonic flow.

Figure 2 shows the corresponding well-known flow representation and the exit pressure evolution $p_{e} / p_{0}$ according to the ratio between the back and upstream stagnation pressure, $p_{b} / p_{0}$.

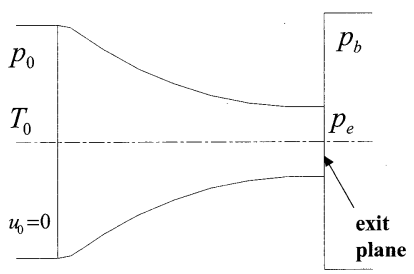

Figure 1: Convergent nozzle and variable definitions.
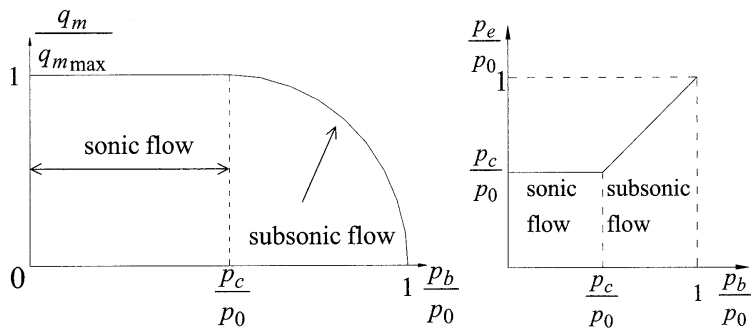

Figure 2: Flow and pressure evolutions for a convergent nozzle.

Flow behaviour in a convergent-divergent nozzle Similarly, the application of fluid mechanic theory to an ideal convergent-divergent nozzle (Fig.3) leads to four main types of flow behaviours. Figure 4 shows flow and pressure changes according to the pressure ratio $p_{b} / p_{0}$.

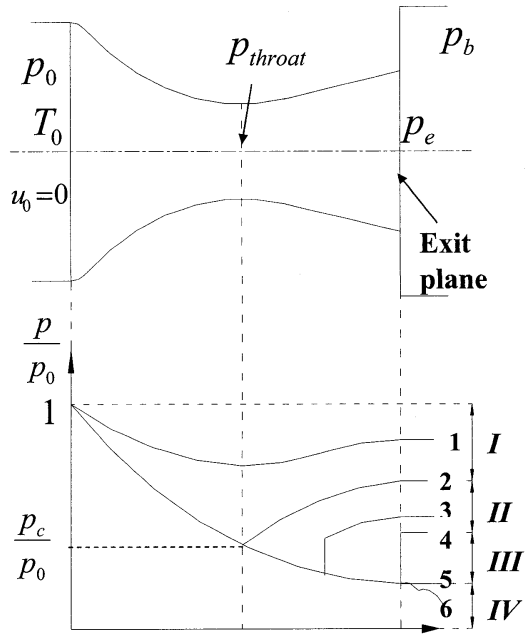

Figure 3: Convergent-divergent nozzle and different pressure distributions according to back pressure value.

The flow curve (Fig.4c) presents two regions corresponding to the subsonic or sonic flow at throat. But downstream the throat, in the divergent part of the nozzle, the flow can be either subsonic or supersonic according to the back pressure value. Furthermore, a normal shock in the divergent section or oblique shocks outside the nozzle outlet can take place. The flow is then no more isentropic downstream to the normal shock in the divergent part of the nozzle. 


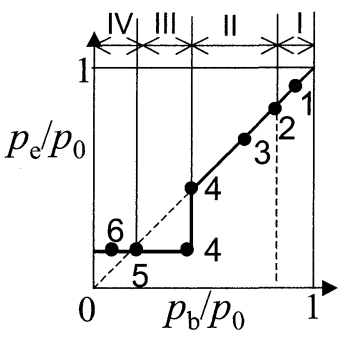

a- Exit pressure

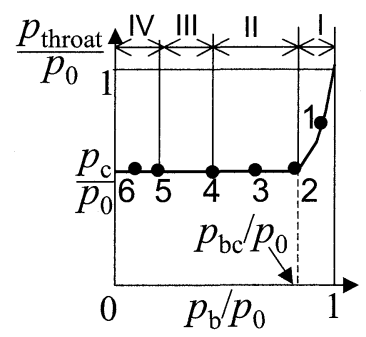

$\mathrm{b}$ - Throat pressure

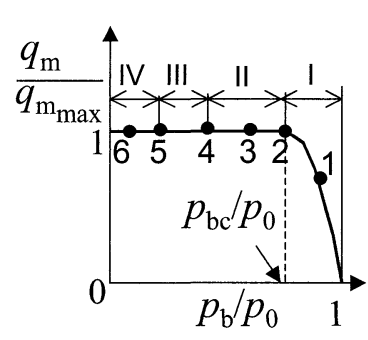

c - Mass flow rate

Figure 4: Characteristics of a convergent-divergent nozzle according to the back to stagnation pressure ratio [7].

For point 4 , the normal shock is located at the exit plane inducing a discontinuity on the pressure curve (Fig.4a).

\section{FLOW CHARACTERIZATION}

Real orifices show some differences with the ideal cases: - There is a contraction of the stream lines at throat: the effective area is smaller than the geometrical one.

- Friction losses are not negligible.

- According to the rate of kinetic energy recovery, the static pressure is higher downstream than in an ideal nozzle.

- The flow can generally be considered as adiabatic (conservation of the total temperature), but it is not isentropic inducing a loss in total pressure.

Experimental results have shown that however, many components have a flow characteristic that is similar to the one obtained for a convergent nozzle (Fig.2). That is why the ISO 6358 standard proposed a mathematical approximation in which the critical pressure ratio is determined experimentally. The subsonic region is approximated by a quarter of ellipse.

But in the case of a convergent-divergent nozzle, this part of the mass flow rate curve corresponds only to the lower part of a quarter of ellipse. An approximation of this characteristic is however possible using the new formula with four parameters [2].

The flow characterisation consists in the measurement of the mass flow rate, and of the upstream and downstream pressures. However the main question is how and where these pressures have to be measured?

According to fluid mechanics, the total upstream pressure has to be considered as well as the exit and back pressures. But the main difficulty is that the exit pressure can not be measured directly because the internal geometry of components is generally complex and the most limiting section is not always located at outlet but often inside the component itself.

In ISO6358:1989 standard, the pressure sensors are installed on measuring tubes that have the same inner diameter as the port of the component to be characterised. This means that the measured upstream and downstream pressures $p_{1 \text { ISO }}$ and $p_{2 \text { ISO }}$ are static pressures. Thus the flow characteristics are depending on the diameter of the connecting tubes because the fluid velocity is not negligible in these tubes. Measurement methods that allow a better correlation with fluid mechanics theory are now explored.

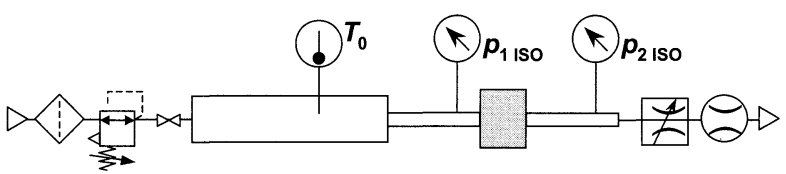

Figure 5 Test bench according to ISO 6358 standard.

\section{UPSTREAM PRESSURE}

In order to obtain flow characteristic parameters independent from the upstream velocity, it is necessary to take into account the total pressure. This pressure can be measured directly on a tube of sufficiently large diameter to have a negligible fluid velocity. It can also be obtained by calculation.

By definition, the total pressure is the pressure the flow would reach if brought isentropically to rest [7]. The equivalent total pressure can then be calculated from the static pressure $p$, with the knowledge of the local geometrical section $A$ :

$p_{t}=p\left[\frac{1}{2}+\sqrt{\frac{\gamma-1}{2 \gamma} R T_{t}\left(\frac{q_{m}}{p A}\right)^{2}+\frac{1}{4}}\right]^{\frac{\gamma}{\gamma-1}}$

In the following, the total pressures are calculated assuming the flow is adiabatic: the local total temperature equals the upstream stagnation temperature $T_{0}$ (Fig.5).

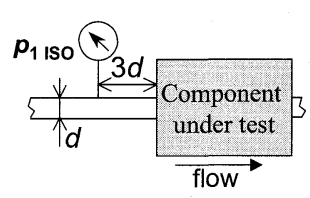

a - ISO 6358:1989.

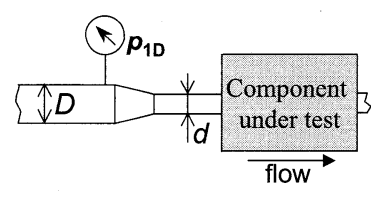

$\mathrm{b}$ - Conical connector.
Figure 6 Upstream pressure connectors used.

In order to measure directly the stagnation pressure, the upstream part of the test bench of ISO 6358 can be modified using the measuring tube shown in Fig.6b. 


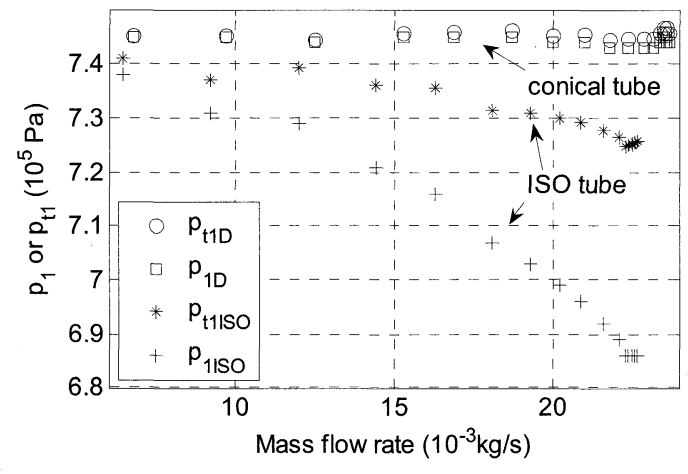

a - Component 1.

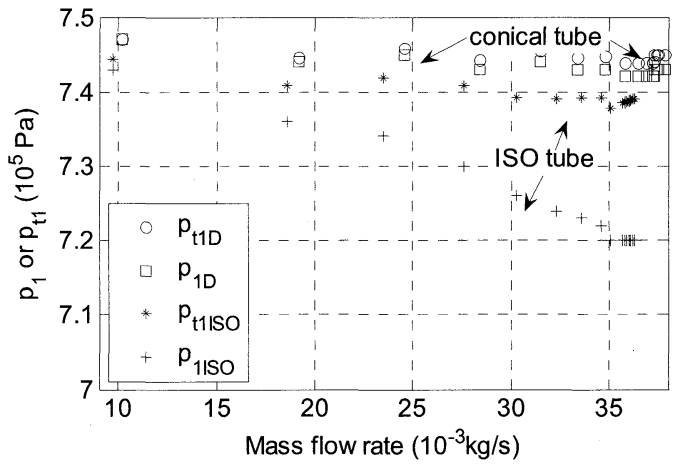

b - Component 2.

Figure 7 Comparison of upstream pressures ( $p_{1}$ measured and $p_{t 1}$ calculated).

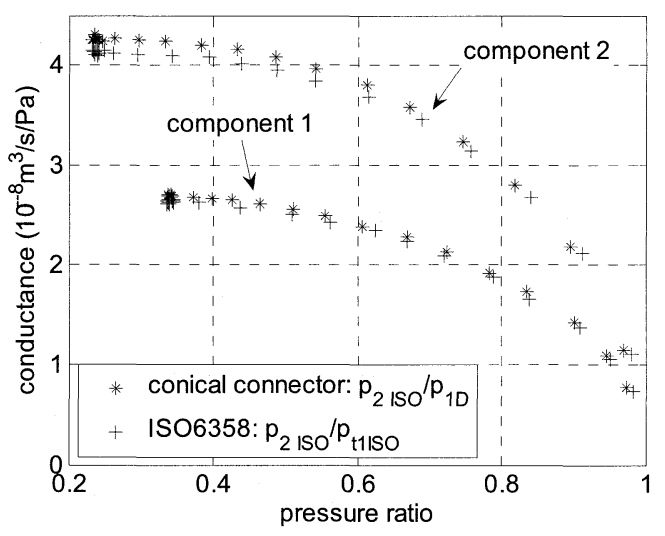

a-Comparison for 2 different total pressures.

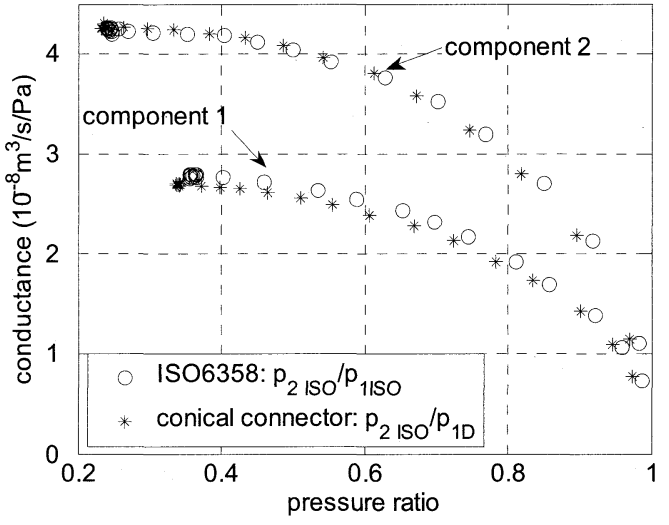

b- Comparison between ISO characteristic and the use of $p_{1 D}$.

Figure 8 Flow characteristics for different upstream pressures (downstream pressure $p_{2 \text { Iso }}$ from ISO 6358 tube).

The upstream pressure is measured on a diameter $D$ two sizes larger than the port size of the component under test. The connection to the component is done with a conical part in order to minimise the losses.

In this section, in order to draw comparisons concerning the upstream pressure, experimental results are obtained using an ISO6358 downstream measuring tube.

Figure 7 shows that for both components, the pressure $p_{1 D}$ measured on the larger diameter of the conical tube is very close to the corresponding calculated upstream total pressure $p_{t 1 D}$ because the velocity of the fluid is negligible. It means that this measured pressure $p_{1 D}$ can be considered as a total pressure. But in the case of the ISO6358 tube, it is clear that the higher the mass flow is, the larger the difference between the calculated total pressure $p_{t 1 \text { Iso }}$ and the measured upstream pressure $p_{1 \mathrm{ISO}}$.

Figure 8 a compares the flow characteristics obtained with the measured total pressure $p_{1 D}$ (on conical tube) and with the calculated total pressure $p_{t 1 \text { ISo }}$ (ISO tube). The conductance is calculated according to ISO6358 standard [1]:

$$
C=\frac{q_{m}}{\rho_{0} p_{1}} \sqrt{\frac{T_{0}}{T_{1}}}
$$

For low conductances in the subsonic range, the characteristics are very close, but when the upstream velocity is higher (especially in ISO tubes) the characteristic obtained with the calculated total pressure $p_{t 1 \text { ISO }}$ is slightly smaller than the one obtained with the direct measurement $p_{1 D}$. It points out the limitation of calculating the total pressure according to (1) due to uncertainties on the effective area $A$ of the flow at the sensor location and on the total temperature $T_{t}$. This means that the direct measurement of the upstream total pressure is preferable using a tube of sufficiently large diameter to make velocity negligible.

Figure $8 \mathrm{~b}$ shows that using this upstream total pressure, the flow characteristic for component 1 is different from the one obtained using the ISO 6358 standard bench (Fig.5). This may seem obvious since the ISO 6358 standard uses a static upstream pressure. However it has to be noticed that for component 2 , both curves are close. This can be explained by lower velocities reached in the ISO 6358 upstream tube than for component 1. 


\section{DOWNSTREAM PRESSURE}

Considering fluid mechanics results, both exit and back pressures seems to be of interest. A conical connector (Fig.9b) can be used to limit the losses (which could appear as a sudden diameter variation) equipped for the measurement of both pressures:

- the pressure $p_{2 d}$ on the port diameter $d$ is measured close to the component outlet at a distance equal to $3 d$. This pressure corresponds to a static pressure, - the pressure $p_{2 D}$ is measured on the largest diameter $D$ at a distance approximately equal to $13 D$ where the flow reaches again a regular shape.

In this section, the flow characterisations use the upstream conical connector (Fig.6b) in order to obtain directly the upstream total pressure $p_{1 D}$ which is kept constant. For both components under test, stationary flow characterisation tests are realised with the both downstream tubes shown in Figure 9.

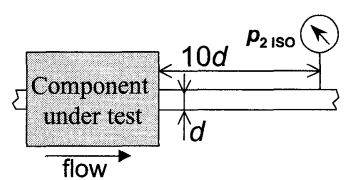

a - ISO 6358:1989.

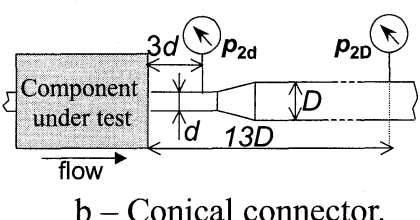

$\mathrm{b}$ - Conical connector.
Figure 9 Downstream pressure connectors.

The use of downstream pressures measured on the component port diameter $d$ either on the ISO6358 tube or on the conical connector leads to the same flow characteristic (Fig.10a). The conical connector presents advantages as it enables lower pressure ratio to be reached during measurement making easier the determination of the characteristic parameters.

The flow characteristic curve is not significantly influenced by the location of pressures measured on the conical connector $p_{2 d}$ or $p_{2 D}$ (Fig.10b). This can be explained by the low losses in subsonic flow between the two pressure sensors locations (Fig.11 and 12).

However the measure of the pressure on the largest diameter $p_{2 D}$ seems preferable because it corresponds to a total pressure (negligible fluid velocity) and thus the complete energy loss is characterised independently of the tubes connected to the component.

The use of the total pressure $p_{t 2 \text { Iso }}$ calculated from the measure of the static pressure $p_{2 \text { ISO }}$ on the ISO 6358 tube of diameter $d$ leads to a different flow characteristic in the case of component 1 (Fig.10c). This component corresponds to a smaller effective section and accordingly higher velocities are reached. It confirms also that the flow is not really isentropic.

Using both downstream pressures measured with the conical connector, the evolution of the pressure ratio $p_{2 d} / p_{1 D}$ versus the ratio of $p_{2 D} / p_{1 D}$ can be plot.

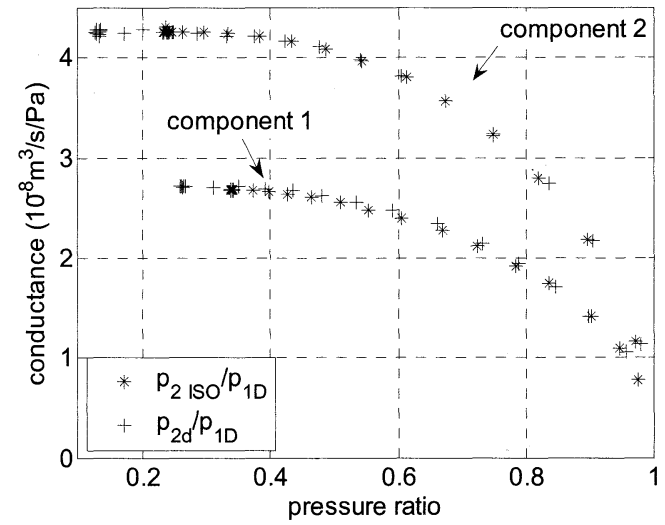

a-Measurements on $d: p_{2 \text { ISO }}$ (ISO6358) and $p_{2 d}$ (conical connector).

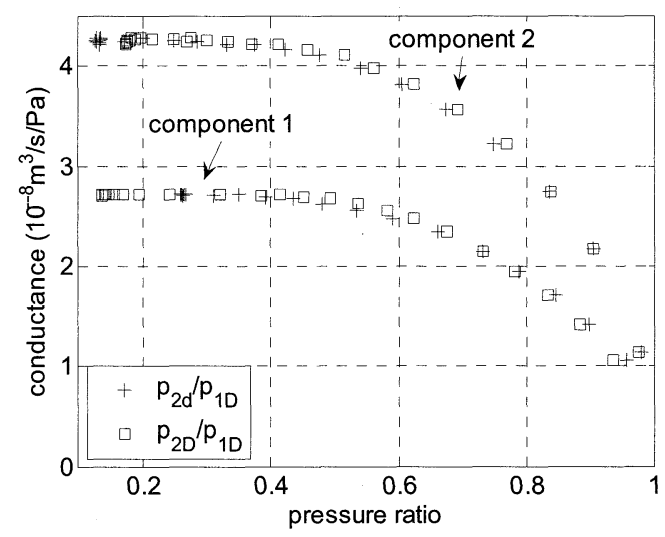

b-On conical connector: $p_{2 d}$ on $d$ and $p_{2 D}$ on $D$.

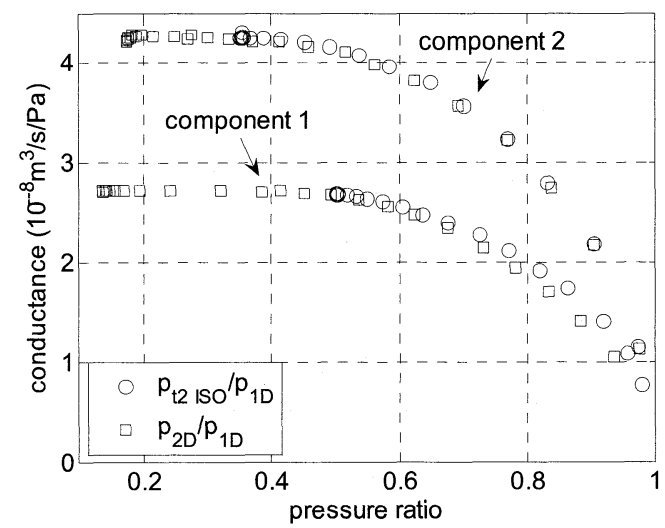

$\mathrm{c}-p_{t 2 \mathrm{ISO}}$ from $p_{2 \mathrm{ISO}}$ on $d$ (ISO6358) and $p_{2 D}$ on $D$ (conical connector).

Figure 10 Flow characteristics of both components according to downstream pressures (upstream pressure $p_{1 D}$ from conical tube).

For component 1 (Fig.11), the form of the curve is similar to the theoretical curve of an ideal convergent nozzle (Fig.2). However, for component 2 (Fig.12), this evolution presents a sudden variation similarly to the case of a convergent-divergent nozzle (Fig.4). 


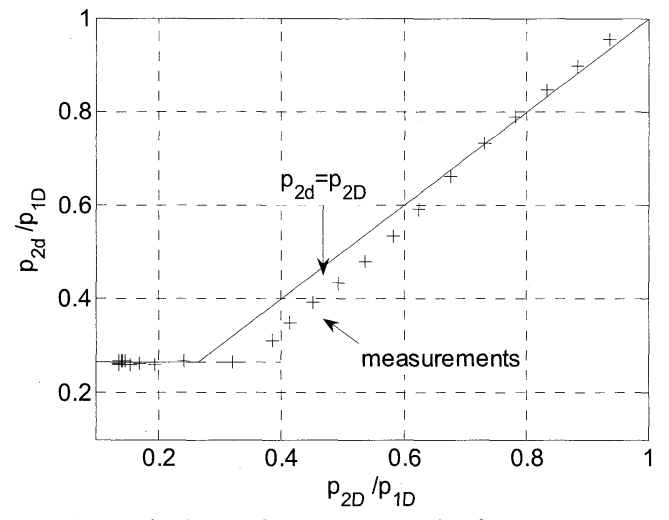

Figure 11 Evolution of pressure ratio for component 1.

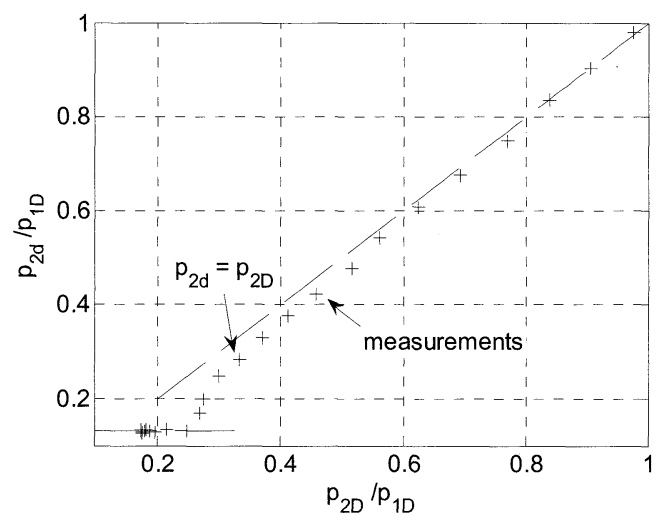

Figure 12 Evolution of pressure ratio for component 2.

In both cases the slope of the oblique line is however different from 1 as in the ideal cases. It shows that there are energy losses between the two downstream pressure sensors. The real flow is not isentropic even with the soft enlargement of the connector. The difference with the ideal cases can come also from the real flow in component which can be tridimensional.

These last remarks mean that the measured pressures can only be considered as an indication respectively of the exit and of the back pressures and not as an accurate measurement of these characteristic pressures.

But it seems sufficient to classify the components under test into two categories according to the similarity of their global flow behaviour with convergent or convergent-divergent ideal nozzles.

\section{CONCLUSION}

Keeping in mind that the standard must help users for the choice of the right component, it is necessary to characterise the global losses of the component independently from the functioning conditions: the considered upstream and downstream pressures should be total pressures. The use of conical connectors with the pressure measurements on the largest diameter enables a direct measurement of the necessary total pressures. The use of total pressures presents also the advantage to make easier the calculations of serial association of components [2, 8].

According to fluid mechanics theory, the additional measurement of the pressure close to the component outlet on a diameter corresponding to the component outlet standard connection can give an indication on the global flow behaviour of the component compared to the ideal cases of convergent or convergent-divergent nozzles. This can be useful at the user level since it is well-known that a convergent-divergent nozzle behaviour means that shocks can take place inside the component or downstream.

The analysis proposed here for stationary flows needs naturally to be validated on other types of components and for transient tests to verify that the diverging part of the downstream connector does not change the flow behaviour of the component due to couplings.

\section{ACKNOWLEDGMENT}

The authors wish to thank the French Technical Centre of Mechanical Industries (CETIM) for its financial support and its collaboration during this work.

\section{REFERENCES}

1. International Standard ISO6358. Pneumatic Fluid Power - Components using Compressible Fluids Determination of Flow-rate Characteristics, 1989. $15 \mathrm{p}$.

2. Yan, T., Guo Xiang, M., Huping, Z., Oneyama, N. Study on Composite Flow-rate Characteristics of Pneumatic Components, Spring symposium on fluid power, JFPS, pp. 101-103, 2003.

3. Kawashima, K., Kagawa, T., Fujita, T., Instantaneous flow rate measurement of ideal gases. Trans. of the ASME Journal of Dynamic Systems, Measurement and Control, vol. 1222, pp.174-178, march 2000.

4. De Giorgi, R., Sesmat, S., Bideaux, E., Using Inverse Models for Determining Orifices Mass Flow Rate Characteristics, Proc. of the $6^{\text {th }}$ JFPS Int. Symp. on Fluid Power, Tsukuba 2005, Nov 7-10, 2005

5. Kuroshita, K., Sekigushi, Y., Oshiki, K., Oneyama, N., Development of new test method for flow-rate characteristics of pneumatic components. In Power Transmission and Motion Control, PTMC'2004, Sept. 2004, Bath, England, pp243-256.

6. F.-E. Sanville. New method of specifying the flow capacity of pneumatic fluid power valves. Hydraulic and pneumatic power, ${ }^{\circ} 195$, vol. 17, mars 1971.

7. White F., M., Fluid Mechanics, McGraw-Hill, New York, $3^{\text {rd }}$ Ed., 1994, 736p.

8. Sesmat, S., Lecerf, J.-P., Gautier, D., Hubert, D., Bideaux, E., Lallement, J., Calculation method for determining the flow-rate characteristics of a group of pneumatic components, Hydraulics and pneumatics'2007, Wroclaw, 10-12 Oct. 2007, pp.409-419 